\title{
On bi-hamiltonian geometry of some integrable systems on the sphere with cubic integral of motion.
}

\author{
A V Vershilov and A V Tsiganov \\ St.Petersburg State University, St.Petersburg, Russia \\ e-mail: alexander.vershilov@gmail.com and tsiganov@mph.phys.spbu.ru
}

\begin{abstract}
We obtain bi-Hamiltonian structure for a family of integrable systems on the sphere $\mathcal{S}^{2}$ with an additional integral of third order in momenta. These results are applied to the Goryachev system and Goryachev-Chaplygin top for which we give an explicit procedure to find the separated coordinates and the separated relations.
\end{abstract}

PACS: $45.10 . \mathrm{Na}, 45.40 . \mathrm{Cc}$

MSC: 70H20; 70H06; 37K10

\section{Introduction}

We address the problem of the separation of variables for the Hamilton-Jacobi equation within the theoretical scheme of bi-hamiltonian geometry. We want to learn to calculate bi-hamiltonian structure for a given integrable system on the Poisson manifold $M$ with the Poisson bivector $P$ and the Casimir functions $C_{a}$. The separation variables are naturally associated with this bi-hamiltonian structure of $M$ itself 6 .

According to [11, 12, 13, we will suppose that desired second Poisson bivector is the Lie derivative of $P$ along some unknown Liouville vector field $X$ on $M$

$$
P^{\prime}=\mathcal{L}_{X}(P)
$$

which has to satisfy to the equation

$$
\left[P^{\prime}, P^{\prime}\right]=\left[\mathcal{L}_{X}(P), \mathcal{L}_{X}(P)\right]=0, \quad \Leftrightarrow \quad\left[\mathcal{L}_{X}^{2}(P), P\right]=0,
$$

with respect to the Schouten bracket [.,.]. By definition (1.1) bivector $P^{\prime}$ is compatible with a given bivector $P$, i.e. $\left[P, P^{\prime}\right]=0$. In geometry such bivector $P^{\prime}$ is said to be the 2 -coboundary associated with the Liouville vector field $X$ in the Poisson-Lichnerowicz cohomology defined by $P$ on $M$.

From all the solutions $X$ of the equation (1.2) we have to choose partial solutions $X$ such that

$$
\left\{H_{i}, H_{j}\right\}^{\prime}=0, \quad i, j=1, \ldots, n,
$$

where $H_{j}$ are the given integrals of motion and $\{., .\}^{\prime}$ is the Poisson bracket associated with the Poisson bivector $P^{\prime}$ (1.1).

Obviously enough, in their full generality the system of equations (1.2 1.3. is too difficult to be solved because it has infinitely many solutions labeled by different separated coordinates, see [13. In order to get particular solutions we will use addition assumptions

$$
P^{\prime} d C_{a}=0, \quad a=1, \ldots, k,
$$

and some special ansätze for the Liouville vector field $X$.

Equations (1.4) say that we are looking for the Poisson bivector $P^{\prime}$, which has the same foliation by symplectic leaves as $P$. In general bivector $P^{\prime}$ could have some more Casimirs, so that their symplectic leaf could be smaller, but in the separation of variables method we have to consider equivalent foliations only [11, 12, 13]. 


\section{A family of integrable systems on the sphere}

A Hamiltonian system is called natural if its Hamiltonian is the sum of a positive-definite kinetic energy and a potential. Natural Hamiltonian systems on cotangent bundles of closed surfaces admitting integrals polynomial in momenta are especially interesting [3. In this Section we define some family of natural Hamiltonian systems on $T^{*} \mathcal{S}^{2}$ with the quadratic and cubic in the momenta integrals of motion.

Let two vectors $J=\left(J_{1}, J_{2}, J_{3}\right)$ and $x=\left(x_{1}, x_{2}, x_{3}\right)$ are coordinates on the Euclidean algebra $e^{*}(3)$ with the Lie-Poisson bracket

$$
\left\{J_{i}, J_{j}\right\}=\varepsilon_{i j k} J_{k}, \quad\left\{J_{i}, x_{j}\right\}=\varepsilon_{i j k} x_{k}, \quad\left\{x_{i}, x_{j}\right\}=0,
$$

where $\varepsilon_{i j k}$ is the totally skew-symmetric tensor. This bracket has two Casimir functions

$$
C_{1}=|x|^{2} \equiv \sum_{k=1}^{3} x_{k}^{2}, \quad C_{2}=(x, J) \equiv \sum_{k=1}^{3} x_{k} J_{k} .
$$

Fixing their values one gets a generic symplectic leaf of $e^{*}(3)$

$$
\mathcal{O}_{A B}: \quad\left\{x, J: C_{1}=A, C_{2}=B\right\},
$$

which is a four-dimensional symplectic manifold.

At $C_{2}=0$ this symplectic manifold is equivalent to cotangent bundle $T^{*} \mathcal{S}^{2}$ of the sphere

$$
\mathcal{S}^{2}=\left\{x \in \mathbb{R}^{3}, \quad|x|=A\right\} .
$$

For the Liouville integrability of the equations of motion it is enough to find one additional integral of motion $H_{2}$, which is functionally independent of the Hamiltonian $H_{1}$ and the Casimir functions.

In this $e^{*}(3)$ coordinates the family of integrable systems on the sphere is defined by the following quadratic Hamiltonian

$$
H_{1}=J_{1}^{2}+J_{2}^{2}+\left(3 \alpha^{2}+f\left(x_{3}\right)\right) J_{3}^{2}+m\left(x_{3}\right) x_{1}+g\left(x_{3}\right),
$$

and cubic additional integral of motion

$$
H_{2}=-2 \alpha J_{3}\left(-\alpha^{2} J_{3}^{2}+J_{1}^{2}+J_{2}^{2}+f\left(x_{3}\right) J_{3}^{2}+g\left(x_{3}\right)\right)-n\left(x_{3}\right) J_{1}-\ell\left(x_{3}\right) x_{1} J_{3} .
$$

Here

$$
\begin{array}{r}
g\left(x_{3}\right)=\frac{a^{2} b}{n\left(x_{3}\right)^{2}}, \quad m\left(x_{3}\right)=-\frac{n^{\prime}\left(x_{3}\right)}{\alpha}, \quad \ell\left(x_{3}\right)=\frac{n\left(x_{3}\right) n^{\prime \prime}\left(x_{3}\right)}{n^{\prime}\left(x_{3}\right)}, \\
f\left(x_{3}\right)=1-3 \alpha^{2}-\alpha \frac{3 x_{3} m\left(x_{3}\right)-2\left(A^{2}-x_{3}^{2}\right) m^{\prime}\left(x_{3}\right)}{n\left(x_{3}\right)}+\frac{x_{3} \ell\left(x_{3}\right)-\left(A^{2}-x_{3}^{2}\right) \ell^{\prime}\left(x_{3}\right)}{n\left(x_{3}\right)},
\end{array}
$$

and

$$
n\left(x_{3}\right)=-a\left(x_{3}+c\right)^{\beta}, \quad a, b, c \in \mathbb{R} .
$$

At $C_{2}=0$ integrals of motion $H_{1}(2.3)$ and $H_{2}(2.4)$ are in the involution with respect to the 
brackets (2.1) in the following five cases:

$$
\begin{array}{llll}
\text { 1. } \alpha=\beta=1, & c=0, & n\left(x_{3}\right)=-a x_{3}, \\
\text { 2. } \alpha=\beta=\frac{1}{3}, & c=0, & n\left(x_{3}\right)=-a x_{3}^{1 / 3}, \\
\text { 3. } \alpha=\beta=\frac{1}{6}, & c=A, & n\left(x_{3}\right)=-a\left(x_{3}+A\right)^{1 / 6}, \\
\text { 4. } \alpha=\beta=\frac{1}{2}, & c \in \mathbb{R}, & n\left(x_{3}\right)=-a\left(x_{3}+c\right)^{1 / 2}, \\
\text { 5. } \alpha=\beta=\frac{1}{4}, & c=A, & n\left(x_{3}\right)=-a\left(x_{3}+A\right)^{1 / 4}, .
\end{array}
$$

The corresponding Hamiltonian (2.3) depends on parameters $a, b$ and $c$ :

$$
\begin{aligned}
& H_{1}^{(1)}=J_{1}^{2}+J_{2}^{2}+4 J_{3}^{2}+a x_{1}+\frac{b}{x_{3}^{2}} \\
& H_{1}^{(2)}=J_{1}^{2}+J_{2}^{2}+\frac{4}{3} J_{3}^{2}+\frac{a x_{1}}{x_{3}^{2 / 3}+\frac{b}{x_{3}^{2 / 3}}} \\
& H_{1}^{(3)}=J_{1}^{2}+J_{2}^{2}+\left(\frac{7}{12}+\frac{x_{3}}{2\left(x_{3}+A\right)}\right) J_{3}^{2}+\frac{a x_{1}}{\left(x_{3}+A\right)^{5 / 6}}+\frac{b}{\left(x_{3}+A\right)^{1 / 3}} \\
& H_{1}^{(4)}=J_{1}^{2}+J_{2}^{2}+\left(1+\frac{x_{3}}{x_{3}+c}-\frac{x_{3}^{2}-c^{2}}{4\left(x_{3}+c\right)^{2}}\right) J_{3}^{2}+\frac{a x_{1}}{\left(x_{3}+c\right)^{1 / 2}}+\frac{b}{x_{3}+c} \\
& H_{1}^{(5)}=J_{1}^{2}+J_{2}^{2}+\left(\frac{13}{16}+\frac{3 x_{3}}{8\left(x_{3}+A\right)}\right) J_{3}^{2}+\frac{a x_{1}}{\left(x_{3}+A\right)^{3 / 4}}+\frac{b}{\left(x_{3}+A\right)^{1 / 2}}
\end{aligned}
$$

The Hamilton function $H_{1}^{(1)}$ describes the well-studied Goryachev-Chaplygin top [1]. In contrast with this case the other systems have not at all physical meaning, however they may be interesting as some mathematical toys. The second integrable system with the Hamiltonian $H_{1}^{(2)}$ was found by Goryachev [2]. The Hamilton function $H_{1}^{(4)}$ was studied by Dullin and Matveev [4]. The third and fifth integrable systems with Hamiltonians $H_{1}^{(3)}$ and $H_{1}^{(5)}$ have been found in 8 .

\section{Construction of the Liouville vector field}

In this Section we describe how to obtain desired Liouville vector field $X$ and the corresponding second Poisson bivector $P^{\prime}$ for our family of integrable systems. Remind, that smooth manifold $M$ endowed with a pair of compatible Poisson bivectors $P$ and $P^{\prime}$ is said to be $\omega N$ manifold if one of the Poisson brackets is non degenerate [6].

Without loss of generality we can always put $|x|=A=1$ by using scaling transformation $x_{j} \rightarrow A^{-1} x_{j}$ and consider the unit sphere $\mathcal{S}^{2}$ only. On its cotangent bundle $T^{*} \mathcal{S}^{2}$ we introduce the following coordinates

$$
\phi=\arctan \left(\frac{x_{1}}{x_{2}}\right), \quad u=x_{3}, \quad p_{\phi}=-J_{3}, \quad p_{u}=\frac{J_{1} x_{2}-x_{1} J_{2}}{x_{1}^{2}+x_{2}^{2}}
$$


so that

$$
\begin{array}{ll}
J_{1}=\frac{u}{\sqrt{1-u^{2}}} \sin (\phi) p_{\phi}+\sqrt{1-u^{2}} \cos (\phi) p_{u}, & x_{1}=\sqrt{1-u^{2}} \sin (\phi), \\
J_{2}=\frac{u}{\sqrt{1-u^{2}}} \cos (\phi) p_{\phi}-\sqrt{1-u^{2}} \sin (\phi) p_{u}, & x_{2}=\sqrt{1-u^{2}} \cos (\phi), \\
J_{3}=-p_{\phi}, \quad x_{3}=u . &
\end{array}
$$

In these coordinates $u, \phi, p_{u}, p_{\phi}$ initial Poisson tensor $P$ associated with the brackets (2.1) becomes canonical tensor on 2-dimensional symplectic manifold

$$
P=\left(\begin{array}{cccc}
0 & 0 & 1 & 0 \\
0 & 0 & 0 & 1 \\
-1 & 0 & 0 & 0 \\
0 & -1 & 0 & 0
\end{array}\right)
$$

which is non degenerate.

Calculations in these coordinates are faster than calculations in the standard spherical coordinates $\phi, \theta=\arccos u$. Of course, using these coordinates on symplectic leaf of $e^{*}(3)$ we implicitly accepted assumptions (1.4) for the initial Poisson manifold $e^{*}(3)$ with the Casimir functions (2.2).

The Hamiltonian (2.3) in these coordinates looks like

$$
\begin{aligned}
H_{1} & =\left(1-u^{2}\right) p_{u}^{2}+\left(3 \alpha^{2}+f(u)+\frac{u^{2}}{1-u^{2}}\right) p_{\phi}^{2}+\sqrt{1-u^{2}} \sin (\phi) m(u)+g(u) \\
& =\mathrm{g}_{u} p_{u}^{2}+\mathrm{g}_{\phi} p_{\phi}^{2}+V(u, \phi),
\end{aligned}
$$

where

$$
\mathrm{g}_{u}=\left(1-u^{2}\right), \quad \mathrm{g}_{\phi}=3 \alpha^{2}+f(u)+\frac{u^{2}}{1-u^{2}} .
$$

According to 9 in order to solve equations $(1.2+1.3)$ we will use polynomial in momenta ansätze for the components of the Liouville vector field $X=\sum X^{j} \partial_{j}$ :

$$
X^{j}=\sum_{k=0}^{N} \sum_{m=0}^{k} y_{k m}^{j}(u, \phi) p_{u}^{k-m} p_{\phi}^{m} .
$$

For all the systems we put $N=2$, it means that $X^{j}$ will be generic second order polynomials in momenta $p_{u}, p_{\phi}$ with coefficients $y_{k m}^{j}(u, \phi)$ depending on variables $u$ and $\phi$.

Substituting this ansatz (3.3) into the equations (1.2,1.3) and demanding that all the coefficients at powers of $p_{u}$ and $p_{\phi}$ vanish one gets the over determined system of 60 algebrodifferential equations on the 24 functions $y_{k m}^{j}(u, \phi)$ which can be easily solved in the modern computer algebra systems.

Below we discuss all the obtained solutions.

\subsection{Particular solution}

For the Goryachev-Chaplygin top [1] with integral of motion $H_{1,2}^{(1)}$ one get the following

Proposition 1 At the first case $\alpha=\beta=1$ and $n\left(x_{3}\right)=a x_{3}$ there is one particular solution 
depending on coordinate $u$ only:

$$
P^{\prime}=\left(\begin{array}{cccc}
0 & u & \frac{u p_{\phi}}{1-u^{2}} & \left(1-u^{2}\right) p_{u} \\
& & \frac{\left(1-2 u^{2}\right) p_{u}}{1-u^{2}} & \frac{\left(2-u^{2}\right) p_{\phi}}{1-u^{2}} \\
* & 0 & 0 & u p_{u}^{2}+\frac{u p_{\phi}^{2}}{1-u^{2}}+\frac{b}{u^{3}} \\
* & * & * & 0
\end{array}\right)
$$

The entries of $P^{\prime}$ are real functions on initial variables.

By construction this Poisson bivector compatible with $P$ (3.1) and functions $H_{1,2}$ are in biinvolution with respect to the corresponding Poisson brackets. So that the phase space $T^{*} \mathcal{S}^{2}$ becomes semisimple $\omega N$ manifold and the foliation defined by $H_{1,2}$ is separable in the DarbouxNijenhuis variables [6].

The variables of separation $q_{1,2}$ (the Darboux-Nijenhuis variables) are the eigenvalues of the recursion operator $N=P^{\prime} P^{-1}$. They are simply roots of the following minimal characteristic polynomial of $N$

$$
\begin{aligned}
\mathcal{A}(\lambda)=(\operatorname{det}(N-\lambda \mathrm{I}))^{1 / 2} & =\lambda^{2}-2 p_{\phi} \lambda-\left(1-u^{2}\right) p_{u}^{2}-\frac{u^{2} p_{\phi}^{2}}{1-u^{2}}-\frac{b}{u^{2}}= \\
& =\lambda^{2}+2 J_{3} \lambda-J_{1}^{2}-J_{2}^{2}-\frac{b}{x_{3}^{2}} .
\end{aligned}
$$

At $b=0$ these variables of separation have been found by Chaplygin in [1]. In initial $e^{*}(3)$ variables this bivector

$$
P^{\prime}=\left(\begin{array}{cccccc}
0 & -x_{3}^{2} & x_{3} x_{2} & -x_{2} J_{1} & -x_{2} J_{2} & x_{3} J_{2}-2 x_{2} J_{3} \\
* & 0 & -x_{3} x_{1} & x_{1} J_{1} & x_{1} J_{2} & 2 x_{1} J_{3}-x_{3} J_{1} \\
* & * & 0 & 0 & 0 & -x_{1} J_{2}+x_{2} J_{1} \\
* & * & * & 0 & -J_{1}^{2}-J_{2}^{2} & -J_{3} J_{2} \\
* & * & * & * & 0 & J_{1} J_{3} \\
* & * & * & * & * & 0
\end{array}\right)
$$

has been found in [10. It is easy to prove that $P^{\prime}$ has the same foliations by symplectic leaves as $P$ at $C_{2}=0$.

\subsection{Generic solution}

Using quadratic in momenta ansatz (3.3) one got the generic solution depending on a pair of parameters $\mu$ and $\nu$ for all five cases of integrable systems (2.8).

Proposition 2 For integrable system (2.3 2.4) equations (1.2 1.3) have the following solution

$$
P_{\mu \nu}^{\prime}=\left(\begin{array}{cccc}
0 & \mathcal{X} & \mathcal{Y}+4 \mathrm{i} \alpha \mu p_{\phi} & 0 \\
* & 0 & \mathcal{Z} & 4 \mathrm{i} \alpha \mu p_{\phi} \\
* & * & 0 & \mathcal{X} \frac{\mathrm{i} m(u) \mathrm{e}^{\mathrm{i} \phi}}{2 \sqrt{1-u^{2}}} \\
* & * & * & 0
\end{array}\right),
$$


where

$$
\begin{aligned}
& \mathcal{X}=-2 \mathrm{i} \mu(u+d)+\frac{\nu}{n(u) m(u)}, \quad \mathrm{i}=\sqrt{-1}, \\
& \mathcal{Y}=\mathcal{X}\left[\mathrm{i} p_{u}+\left(\frac{3 \alpha-1}{u+c}-\frac{u}{1-u^{2}}\right) p_{\phi}\right], \\
& \mathcal{Z}=\frac{\left(-2 \mathrm{i} \mu a^{2}(u+d)^{2 \beta}-\nu\right)}{n(u)^{2}}\left[\left(\alpha-\frac{u(u+c)}{\left(1-u^{2}\right)}\right) p_{u}-\frac{\mathrm{i}(u+c) \mathrm{g}_{\phi}}{\left(1-u^{2}\right)} p_{\phi}\right],
\end{aligned}
$$

Here $\mathrm{g}_{\phi}$ is a component of metric (3.2) and

- $d=0$ at cases 1,2,4,

- $d=A$ at cases 3,5.

The complex conjugated bivector $\overline{P^{\prime}}{ }_{\mu \nu}$ is another solution of the same equations (1.2]1.3).

In contrast with the particular solution (3.4) entries of this Poisson bivector are complex functions on initial variables and $P_{\mu \nu}^{\prime}$ depends on parameter $a$ instead of parameter $b$.

As above this Poisson bivector is compatible with $P$ (3.1) and functions $H_{1,2}$ are in biinvolution with respect to the corresponding Poisson brackets. So, the phase space $T^{*} \mathcal{S}^{2}$ becomes semisimple $\omega N$ manifold and the foliation defined by $H_{1,2}$ is separable in the DarbouxNijenhuis variables, which are the eigenvalues of the recursion operator $N=P^{\prime} P^{-1}$ [6].

Summing up, we have found particular and generic solutions of the equations (1.2 1.3) for five integrable systems (2.8) on the sphere with cubic integrals of motion. An important application of this result is the separation of variables for these systems.

\section{Separation of variables}

In this section we consider new separated variables and separated relations for the Goryachev system [2] and for the Goryachev-Chaplygin top [1] in details.

\subsection{The Goryachev system}

In this case

$$
\alpha=\beta=\frac{1}{3}, \quad n=-a x_{3}^{1 / 3}, \quad m=\frac{a}{x_{3}^{2 / 3}}, \quad \ell=\frac{2 a}{3 x_{3}^{2 / 3}}, \quad g=\frac{b}{x_{3}^{2 / 3}}, \quad f=1,
$$

so that

$$
H_{1}^{(2)}=J_{1}^{2}+J_{2}^{2}+\frac{4}{3} J_{3}^{2}+\frac{a x_{1}}{x_{3}^{2 / 3}}+\frac{b}{x_{3}^{2 / 3}}
$$

and

$$
H_{2}^{(2)}=-\frac{2}{3} J_{3}\left(J_{1}^{2}+J_{2}^{2}+\frac{8}{9} J_{3}^{2}+\frac{b}{x_{3}^{2 / 3}}\right)+a x_{3}^{1 / 3} J_{1}-\frac{2 a x_{1} J_{3}}{3 x_{3}^{2 / 3}} .
$$

If we put $\mu=0$ and $\nu=-a^{2}$ in $P_{\mu \nu}^{\prime}$ (3.7) one gets the following second bivector on $T^{*} \mathcal{S}^{2}$ :

$$
P^{\prime}=\left(\begin{array}{cccc}
0 & u^{1 / 3} & \mathrm{i} u^{1 / 3} p_{u}-\frac{u^{4 / 3}}{1-u^{2}} p_{\phi} & 0 \\
* & 0 & \frac{1-4 u^{2}}{3 u^{2 / 3}\left(1-u^{2}\right)} p_{u}+\frac{\mathrm{i} u^{1 / 3}\left(4-u^{2}\right)}{3\left(1-u^{2}\right)^{2}} p_{\phi} & 0 \\
* & * & 0 & \frac{\mathrm{i} a \mathrm{e}^{\mathrm{i} \phi}}{2 u^{1 / 3} \sqrt{1-u^{2}}} \\
* & * & * & 0
\end{array}\right)
$$


The variables of separation $q_{1,2}$ (the Darboux-Nijenhuis variables) are eigenvalues of the recursion operator $N=P^{\prime} P^{-1}$, which are roots of the following polynomial

$$
\mathcal{A}(\lambda)=\left(\lambda-q_{1}\right)\left(\lambda-q_{2}\right)=\lambda^{2}+u^{1 / 3}\left(\frac{u p_{\phi}}{1-u^{2}}-\mathrm{i} p_{u}\right) \lambda-\frac{\mathrm{i} a \mathrm{e}^{\mathrm{i} \phi}}{2 \sqrt{1-u^{2}}} .
$$

In the initial $e^{*}(3)$ variables the second Poisson brackets look like

$$
\begin{aligned}
\left\{x_{i}, x_{j}\right\}^{\prime} & =\varepsilon_{i j k} x_{k} x_{3}^{1 / 3}, \quad\left\{x_{j}, J_{3}\right\}^{\prime}=0 \\
\left\{x_{1}, J_{1}\right\}^{\prime} & =\frac{x_{2} J_{1}}{3 x_{3}^{2 / 3}}-\frac{x_{3}^{4 / 3} J_{2}}{x_{1}+\mathrm{i} x_{2}}+\frac{4 x_{3}^{1 / 3} x_{2} J_{3}}{3\left(x_{1}+\mathrm{i} x_{2}\right)} \\
\left\{x_{2}, J_{2}\right\}^{\prime} & =\frac{\mathrm{i} x_{3}^{4 / 3} J_{1}}{x_{1}+\mathrm{i} x_{2}}-\frac{x_{1} J_{2}}{3 x_{3}^{2 / 3}}-\frac{4 \mathrm{i} x_{3}^{1 / 3} x_{1} J_{3}}{3\left(x_{1}+\mathrm{i} x_{2}\right)} \\
\left\{x_{1}, J_{2}\right\}^{\prime} & =\frac{\mathrm{i}\left(x_{2}^{2}-\mathrm{i} x_{1} x_{2}-3 x_{3}^{2}\right) J_{2}}{3 x_{3}^{2 / 3}\left(x_{1}+\mathrm{i} x_{2}\right)}+\frac{4 \mathrm{i} x_{3}^{1 / 3} x_{2} J_{3}}{3\left(x_{1}+\mathrm{i} x_{2}\right)} \\
\left\{x_{2}, J_{2}\right\}^{\prime} & =-\frac{\left(x_{1}^{2}+\mathrm{i} x_{1} x_{2}-3 x_{3}^{2}\right) J_{1}}{3 x_{3}^{2 / 3}\left(x_{1}+\mathrm{i} x_{2}\right)}-\frac{4 x_{3}^{1 / 3} x_{1} J_{3}}{3\left(x_{1}+\mathrm{i} x_{2}\right)} \\
\left\{x_{3}, J_{1}\right\}^{\prime} & =-\frac{x_{3}^{1 / 3}\left(J_{1} x_{2}-x_{1} J_{2}\right)}{x_{1}+\mathrm{i} x_{2}}, \quad\left\{x_{3}, J_{2}\right\}^{\prime}=-\frac{\mathrm{i} x_{3}^{1 / 3}\left(J_{1} x_{2}-x_{1} J_{2}\right)}{x_{1}+\mathrm{i} x_{2}}, \\
\left\{J_{i}, J_{j}\right\}^{\prime} & =\frac{-a \varepsilon_{i j k} x_{k}}{2 x_{3}^{1 / 3}\left(x_{1}+\mathrm{i} x_{2}\right)}+\delta_{i 1} \delta_{j 2} \frac{\mathrm{i}\left(J_{1} x_{2}-x_{1} J_{2}\right)\left(J_{1}+\mathrm{i} J_{2}\right)}{3 x_{3}^{2 / 3}\left(x_{1}+\mathrm{i} x_{2}\right)} .
\end{aligned}
$$

The corresponding Poisson bivector $P^{\prime}$ is compatible with canonical Poisson bivector on $e^{*}(3)$ and satisfies to equations (1.4), so that $P^{\prime}$ has the same foliations by symplectic leaves as $P$ at $C_{2}=0$.

According to [6] the bi-involutivity of integrals of motion

$$
\left\{H_{1}^{(2)}, H_{2}^{(2)}\right\}=\left\{H_{1}^{(2)}, H_{2}^{(2)}\right\}^{\prime}=0
$$

is equivalent to the existence of non-degenerate control matrix $F$ such that

$$
P^{\prime} d H_{i}^{(2)}=P \sum_{j=1}^{2} F_{i j} d H_{j}^{(2)}, \quad i=1,2 .
$$

In our case the control matrix $F$ reads as

$$
F=\left(\begin{array}{cc}
-\frac{x_{3}^{1 / 3}\left(J_{1}+\mathrm{i} J_{2}\right)}{x_{1}+\mathrm{i} x_{2}}+\frac{2 J_{3}}{3 x_{3}^{2 / 3}} & \frac{1}{x_{3}^{2 / 3}} \\
\frac{-a x_{3}^{2 / 3}}{2\left(x_{1}+\mathrm{i} x_{2}\right)}+\frac{2 x_{3}^{1 / 3}\left(J_{1}+\mathrm{i} J_{2}\right) J_{3}}{3\left(x_{1}+\mathrm{i} x_{2}\right)}-\frac{4 J_{3}^{2}}{9 x_{3}^{2 / 3}} & \frac{-2 J_{3}}{3 x_{3}^{2 / 3}}
\end{array}\right) .
$$

The Darboux-Nijenhuis variables $q_{1,2}$ are simultaneously eigenvalues of the recursion operator and eigenvalues the control matrix. In our case they are the roots of the following polynomial

$$
\mathcal{A}(\lambda)=\operatorname{det}(F-\lambda \mathrm{I})=\lambda^{2}+\frac{x_{3}^{1 / 3}\left(J_{1}+\mathrm{i} J_{2}\right)}{x_{1}+\mathrm{i} x_{2}} \lambda+\frac{a}{2\left(x_{1}+\mathrm{i} x_{2}\right)} .
$$

The left eigenvectors of $F$, if suitable normalized, form the Stäckel matrix $S$, which enters into a pair of the separated relations

$$
\sum_{j=1}^{2} S_{i j}\left(q_{i}, p_{i}\right) H_{j}^{(2)}-U_{i}\left(q_{i}, p_{i}\right)=0, \quad i=1,2 .
$$


Here $U_{i}$ are the Stäckel potentials and $p_{1,2}$ are variables conjugated to $q_{1,2}$ :

$$
\left\{q_{i}, p_{j}\right\}=\delta_{i j}, \quad\left\{q_{i}, p_{j}\right\}^{\prime}=\delta_{i j} q_{i}, \quad\left\{q_{i}, q_{j}\right\}=\left\{q_{i}, q_{j}\right\}^{\prime}=\left\{p_{i}, p_{j}\right\}=\left\{p_{i}, p_{j}\right\}^{\prime}=0 .
$$

Unfortunately, construction of the variables $p_{1,2}$ is non-algorithmic procedure, which depends on the fortune and skilfulness 6 . In our case we can observe that

$$
q_{1}+q_{2}=\mathrm{i} u^{1 / 3} p_{u}-\frac{u^{4 / 3} p_{\phi}}{1-u^{2}}, \quad \text { and } \quad q_{1} q_{2}=-\frac{\mathrm{i} a \mathrm{e}^{\mathrm{i} \phi}}{2 \sqrt{1-u^{2}}}
$$

so that

$$
\left\{p_{\phi}, q_{1}+q_{2}\right\}=0, \quad\left\{p_{\phi}, q_{1} q_{2}\right\}=-\mathrm{i} q_{1} q_{2}, \quad\left\{u, q_{1}+q_{2}\right\}=\mathrm{i} u^{1 / 3}, \quad\left\{u, q_{1} q_{2}\right\}=0 .
$$

Integrating these equations with respect to $p_{\phi}(q, p)$ and $u(q, p)$ we can easily get the following expressions for these functions

$$
p_{\phi}=\frac{\mathrm{i} q_{1} q_{2}\left(p_{2}-p_{1}\right)}{q_{1}-q_{2}}, \quad \text { and } \quad u=\left(\frac{-2 \mathrm{i}\left(q_{1} p_{1}-q_{2} p_{2}\right)}{3\left(q_{1}-q_{2}\right)}\right)^{3 / 2}
$$

which yield the necessary definitions of the momenta

$$
p_{i}=\mathcal{B}\left(\lambda=q_{i}\right), \quad i=1,2, \quad \text { where } \quad \mathcal{B}(\lambda)=\mathrm{i}\left(\frac{3 u^{2 / 3}}{2}-\frac{p_{\phi}}{\lambda}\right)=\mathrm{i}\left(\frac{3 x_{3}^{2 / 3}}{2}+\frac{J_{3}}{\lambda}\right) .
$$

Using the following relations for the second Poisson bracket

$$
\left\{p_{\phi}, q_{1}+q_{2}\right\}^{\prime}=q_{1} q_{2}, \quad \text { and } \quad\left\{p_{\phi}, q_{1} q_{2}\right\}^{\prime}=0
$$

it is easy to prove that $\left\{q_{i}, p_{j}\right\}^{\prime}=\delta_{i j} q_{i}$.

In the separated variables the Stäckel matrix $S$ is equal to

$$
S=\left(\begin{array}{cc}
1 & 1 \\
\frac{3 \mathrm{i}}{2 q_{1} p_{1}} & \frac{3 \mathrm{i}}{2 q_{2} p_{2}}
\end{array}\right)=\left(\begin{array}{cc}
1 & 1 \\
\frac{-1}{2 \mathrm{i} \alpha q_{1} p_{1}} & \frac{-1}{2 \mathrm{i} \alpha q_{2} p_{2}}
\end{array}\right)
$$

whereas integrals of motion have the generalized Stäckel form at the second case (2.8)

$$
H_{i}^{(2)}=\sum_{j=1}^{2} S_{i j}^{-1}\left(\frac{\left(4 p_{j}^{2}+6 \mathrm{i} p_{j}-9\right)\left(3 \mathrm{i}-2 p_{j}\right) q_{j}^{2}}{18 p_{j}}-\frac{3 \mathrm{i} a^{2}}{8 q_{j}^{2} p_{j}}+\frac{3 \mathrm{i} b}{2 p_{j}}\right), \quad i=1,2 .
$$

The corresponding separated relations (4.3) define two copies of the following algebraic curve

$$
\mathcal{C}: \quad \mu^{3}-H_{1}^{(2)} \mu+\lambda^{3}=b \lambda-H_{2}^{(2)}-\frac{a^{2}}{4 \lambda}, \quad \mu=2 \text { i } \alpha q_{i} p_{i}, \quad \lambda=q_{i} .
$$

In contrast with the usual Stäckel systems it is non-hyperelliptic curve, which is trigonal algebraic curve. It is interesting that one formally gets the real algebraic curve. But we have to bear firmly in mind that our separated variables $q_{1,2}$ and $p_{1,2}$ are complex functions on initial physical variables.

If we put $\nu=0$ and $\mu=1$ in $P_{\mu \nu}^{\prime}$ (3.7) one gets another Poisson bivector on $T^{*} \mathcal{S}^{2}$ :

$$
P^{\prime}=\left(\begin{array}{cccc}
0 & u & \mathrm{i} u p_{u}-\frac{2+u^{2}}{3\left(1-u^{2}\right)} p_{\phi} & 0 \\
* & 0 & \frac{1-4 u^{2}}{3\left(1-u^{2}\right)} p_{u}+\frac{\mathrm{i} u\left(4-u^{2}\right)}{3\left(1-u^{2}\right)^{2}} p_{\phi} & -\frac{2}{3} p_{\phi} \\
* & * & 0 & \frac{\mathrm{i} a u^{1 / 3} \mathrm{e}^{\mathrm{i} \phi}}{2 \sqrt{1-u^{2}}} \\
* & * & * & 0
\end{array}\right)
$$


In this case the Darboux-Nijenhuis coordinates are roots of the following polynomial

$$
\mathcal{A}(\lambda)=\lambda^{2}-\left(\mathrm{i} u p_{u}-\frac{4-u^{2}}{3\left(1-u^{2}\right)} p_{\phi}\right)-\frac{\mathrm{i} a u^{4 / 3} \mathrm{e}^{\mathrm{i} \phi}}{2 \sqrt{1-u^{2}}}-\frac{2 \mathrm{i} u}{3} p_{u} p_{\phi}+\frac{2\left(2+u^{2}\right)}{9\left(1-u^{2}\right)} p_{\phi}^{2} .
$$

In term of the previous separated variables this polynomial looks like

$$
\mathcal{A}(\lambda)=\left(\lambda+\frac{2 \mathrm{i}}{3} q_{1} p_{1}\right)\left(\lambda+\frac{2 \mathrm{i}}{3} q_{2} p_{2}\right) .
$$

Now it is easy to prove that in the separated variables $(q, p)$ bivector $P_{\mu \nu}^{\prime}$ (3.7) looks like

$$
P_{\mu \nu}^{\prime}=-2 \mathrm{i} \alpha \mu\left(\begin{array}{cccc}
0 & 0 & q_{1} p_{1} & 0 \\
0 & 0 & 0 & q_{2} p_{2} \\
-q_{1} p_{1} & 0 & 0 & 0 \\
0 & -q_{2} p_{2} & 0 & 0
\end{array}\right)-\frac{\nu}{a^{2}}\left(\begin{array}{cccc}
0 & 0 & q_{1} & 0 \\
0 & 0 & 0 & q_{2} \\
-q_{1} & 0 & 0 & 0 \\
0 & -q_{2} & 0 & 0
\end{array}\right)
$$

For the remaining four systems bivector $P_{\mu, \nu}$ (3.7) has the similar form in the Darboux-Nijenhuis variables $(q, p)$.

\subsection{The Goryachev-Chaplygin top}

Now we briefly discuss new separation of variables for the Goryachev-Chaplygin top [1]. Remind, that in this case the Hamiltonian reads as

$$
H_{1}^{(1)}=J_{1}^{2}+J_{2}^{2}+4 J_{3}^{2}-a x_{1}+\frac{b}{x_{3}^{2}} .
$$

The new separated coordinates are roots of the polynomial

$$
\begin{aligned}
\mathcal{A}(\lambda) & =\lambda^{2}+a^{2}\left(\frac{i p_{u}}{m n}+\frac{3 \alpha p_{\phi}}{m n(u+c)}+\frac{p_{\phi}(u c+1)}{m n(u+c)\left(u^{2}-1\right)}\right) \lambda+\frac{\mathrm{i} a^{4} \mathrm{e}^{\mathrm{i} \phi}}{2 m n^{2} \sqrt{1-u^{2}}} \\
& =\lambda^{2}-\left(\frac{\mathrm{i} p_{u}}{u}+\frac{3 p_{\phi}}{u^{2}}+\frac{p_{\phi}}{u^{2}\left(u^{2}-1\right)}\right) \lambda+\frac{\mathrm{i} a \mathrm{e}^{\mathrm{i} \phi}}{2 u^{2} \sqrt{1-u^{2}}}
\end{aligned}
$$

whereas the conjugated momenta are equal to

$$
p_{i}=\mathcal{B}\left(\lambda=q_{i}\right), \quad \text { where } \quad \mathcal{B}(\lambda)=\mathrm{i}\left(\frac{u^{2}}{2}-\frac{p_{\phi}}{\lambda}\right) .
$$

These separated variables lie on two copies of the following algebraic curve

$$
\mathcal{C}: \quad \mu\left(\mu^{2}+\mu \lambda-H_{1}^{(1)}\right)=b \lambda-H_{2}^{(1)}-\frac{a^{2}}{4 \lambda}, \quad \mu=2 \mathrm{i} \alpha q_{i} p_{i}, \quad \lambda=q_{i} .
$$

As for Goryachev model these separated variables $q_{1,2}$ and $p_{1,2}$ are complex functions on initial physical variables, which lie on the non-hyperelliptic algebraic curve.

Of course, we can repeat similar calculations for the remaining systems and prove that they are related with different trigonal curves as well. As an example, for the Dullin-Matveev system 4] equation of motion are linearized on the following algebraic curve

$$
\mathcal{C}: \quad \mu\left(\mu^{2}-\lambda^{2}-H_{1}^{(4)}\right)=b \lambda-H_{2}^{(4)}-\frac{a^{2}}{4 \lambda}, \quad \mu=2 \mathrm{i} \alpha q_{i} p_{i}, \quad \lambda=q_{i} .
$$

if $c=0$ in the Hamiltonian $H_{1}^{(4)}(2.8)$.

It will be interesting to get solutions of the equations of motion in term of the abelian functions for trigonal curves, as example see [5]. The other open question is construction of the Lax matrices associated with separated variables on trigonal curves. 


\subsection{The Jacobi method}

The Jacobi method consists of construction of the integrable system starting with some known separated variables and arbitrary separated relations. The method was originally formulated by Jacobi when he invented elliptic coordinates and successfully applied them to solve several important mechanical problems, such as the problem of geodesic motion on an ellipsoid, and the problem of planar motion in a force field of two attracting centers. In [7 Jacobi himself wrote: "The main difficulty in integrating a given differential equation lies in introducing convenient variables, which there is no rule for finding. Therefore, we must travel the reverse path and after finding some notable substitution, look for problems to which it can be successfully applied."

In our case this notable substitution is described by the following

Proposition 3 At any $a, c$ and $\alpha$ transformation

$$
\begin{aligned}
u & =\left(\frac{-2 \mathrm{i} \alpha\left(p_{1} q_{1}-q_{2} p_{2}\right)}{q_{1}-q_{2}}\right)^{1 / 2 \alpha}-c, \quad p_{\phi}=\frac{-\mathrm{i} q_{1} q_{2}\left(p_{1}-p_{2}\right)}{q_{1}-q_{2}}, \\
\phi & =-\frac{\mathrm{i}}{2}\left(2 \ln (u+c)(3 \alpha-1)+\ln \left(\frac{4 q_{1}^{2} q_{2}^{2}\left(u^{2}-1\right)}{a^{2}}\right)\right) \\
p_{u} & =\left(\frac{3 \mathrm{i} \alpha}{u+c}+\frac{\mathrm{i}(u c+1)}{(u+c)\left(u^{2}-1\right)}\right) p_{\phi}-\mathrm{i}\left(q_{1}+q_{2}\right)(u+c)^{2 \alpha-1}
\end{aligned}
$$

is canonical transformation.

Now in order to get some integrable system on $T^{*} \mathcal{S}^{2}$ we could take two copies of any algebraic curve $\mathcal{C}$ defined by equation $\Phi\left(\mu, \lambda, H_{1}, H_{2}\right)=0$ and solve the corresponding separated relations

$$
\Phi\left(\mu_{i}, \lambda_{i}, H_{1}, H_{2}\right)=0, \quad \mu_{i}=2 \mathrm{i} \alpha q_{i} p_{i}, \quad \lambda_{i}=q_{i}
$$

with respect to integrals of motion $H_{1}$ and $H_{2}$, which will be in bi-involution [13. As above the main problem is that change of variables (4.8) is the transformation over the complex field $\mathbb{C}$ and if we want to get real functions $H_{1,2}$ on the initial variables on $T^{*} \mathcal{S}^{2}$ we have to start with the very special algebraic curves $\mathcal{C}$, for instance with (4.4|4.6) or (4.7).

Example: Let us consider the following deformation of the algebraic curve (4.6):

$$
\widetilde{\mathcal{C}}: \quad \mu\left(\mu^{2}+\mu \lambda-H_{1}\right)+\rho\left(\frac{\rho}{4}+\mu\right)(\lambda+\mu)=b \lambda-H_{2}-\frac{a^{2}}{4 \lambda} .
$$

Solving the corresponding separated relations with respect to $H_{1,2}$ one gets integrals of motion in the bi-involution. After canonical transformation (4.8) at $c=0$ and $\alpha=1$ we obtain the following complex Hamiltonian

$$
H_{1}=\left(1-u^{2}\right) p_{u}^{2}+\frac{\left(4-3 u^{2}\right) p_{\phi}^{2}}{1-u^{2}}-a \sin (\phi) \sqrt{1-u^{2}}+\frac{b}{u^{2}}+\rho p_{\phi}-\frac{\rho^{2}\left(1-u^{2}\right)}{4 u^{2}}+\frac{\mathrm{i} \rho p_{u}\left(1-u^{2}\right)}{u},
$$

which after an obvious additional shift of momenta (canonical transformation)

$$
p_{u} \rightarrow \tilde{p}_{u}=p_{u}-\frac{\mathrm{i} \rho}{2 u}
$$

becomes the real Hamiltonian for the Goryachev-Chaplygin gyrostat

$$
H_{1}=H_{1}^{(1)}+\rho p_{\phi}=J_{1}^{2}+J_{2}^{2}+4 J_{3}^{2}-\rho J_{3}-a x_{1}+\frac{b}{x_{3}^{2}} .
$$

More complicated deformations of the algebraic curve (4.6), such as

$$
\mu\left(\mu^{2}+\mu \lambda-H_{1}\right)+c_{1} \mu \lambda+c_{2} \lambda^{2}+c_{3} \mu^{2}=b \lambda-H_{2}-\frac{a^{2}}{4 \lambda},
$$

and the corresponding additional shifts of the momenta $p_{u}$ lead to another generalizations of the Goryachev-Chaplygin top, which was obtained in [14].

Of course, we can get similar deformations for the remaining four systems in (2.8) as well. 


\section{Conclusion}

We found two-parametric Poisson bivector (3.7), which is compatible with canonical Poisson bivector on cotangent bundle $T^{*} \mathcal{S}^{2}$ of two-dimensional sphere. The quadratic and cubic integrals of motion (2.3/2.4) for the five integrable systems on the sphere are in bi-involution with respect to the corresponding Poisson brackets.

The eigenvalues of the corresponding recursion operator are the separated coordinates. For the Goryachev system and Goryachev-Chaplygin top we give an explicit formulae for these separated variables and the corresponding separated relations.

The research was partially supported by the RFBR grant.

\section{References}

[1] D.N. Goryachev, On a Motion of a Heavy Rigid Body About a Fixed Point in the Case of $A=B=4 C$, Mat. sbonik kruzhka lyub. mat. nauk, vol. 21, no. 3, pp. 431-438, 1900.

S.A. Chaplygin, A New Partial Solution of the Problem of Rotation of a Heavy Rigid Body About a Fixed Point, Trudy otd. fiz. nauk Mosk. obshch. lyub. estest., v.12, n.1, p.1-4, 1904.

[2] D.N. Goryachev, New cases of integrability of Euler's dynamical equations, Warshav. Univ. Izv., v.3, p.1-15, 1916.

[3] G. Darboux, Leçons sur la Théorie Générale des Surfaces, 1898.

[4] H.R. Dullin, V.S. Matveev, A new integrable system on the sphere, Mathematical Research Letters, v.11, p.715-722, 2004.

[5] J.C. Eilbeck, V.Z. Enolski, S. Matsutani, Y. Ônishi, E. Previato, Abelian functions for trigonal curves of genus three, International Mathematics Research Notices, v.2007, Art.ID 140 (38 pages), 2007.

[6] G. Falqui, M. Pedroni, Separation of variables for bi-Hamiltonian systems, Math. Phys. Anal. Geom., v.6, p.139-179, 2003.

[7] C.G.J. Jacobi, Vorlesungen über Dynamik, Georg Reimer, Berlin, 1866. Jacobi's lectures on dynamics given in Königsberg 1842-1843 published by A. Clebsch.

[8] A.V. Tsiganov, On a family of integrable systems on $\mathcal{S}^{2}$ with a cubic integral of motion, J. Phys. A, Math. Gen. v.38, p.921-927, 2005.

[9] A.V. Tsiganov, On natural bi-hamiltonian systems, Teor. Math. Phys., v.149(2), p.1437$1456,2006$.

[10] A.V. Tsiganov, A family of the Poisson brackets compatible with the Sklyanin bracket, J. Phys. A, v.40, pp.4803-4816, 2007.

[11] A.V. Tsiganov, Separation of variables for a pair of integrable systems on so*(4), Doklady Math., v.76(3), p.839-842, 2007.

[12] A.V. Tsiganov, On bi-hamiltonian structure of some integrable systems on so*(4), J. Nonlinear Math. Phys., v.15(2), p.171-185, 2008.

[13] A.V. Tsiganov, On bi-hamiltonian geometry of the Lagrange top, J. Phys. A: Math. Theor., v.41, 315212 (12pp), 2008.

[14] H.M. Yehia, On certain two-dimensional conservative mechanical systems with a cubic second integral, J. Phys. A: Math. Gen., v.35, pp. 9469-9487, 2002. 